\title{
Los estilos de liderazgo y los estilos de pensamiento en los directivos de Instituciones Educativas
}

\section{Leadership styles and thinking styles in the directors of Educational Institutions}

\author{
Mary Leid Carranza Romero
}

\begin{abstract}
RESUMEN
En la actualidad, la educación peruana tiene como objetivo principal que todos los niños, niñas y adolescentes logren aprender. Esto consiste en desarrollar sus habilidades, capacidades y, por ende, sus competencias para que puedan enfrentarse a las dificultades que se les presenten en la vida diaria. Para ello, el Ministerio de Educación (Minedu en adelante) ha implementado estrategias, y tiene como principales aliados a los docentes y los directivos dentro de las escuelas. El directivo juega un rol muy importante, ya que de él dependerá si se logran o no los objetivos propuestos para el año académico. Antes, los directivos eran considerados solamente como agentes administrativos, alejados de la parte pedagógica. Sin embargo, hoy en día nuestro sistema educativo los ha colocado como líderes pedagógicos, para que sean partícipes directos de las reformas educativas que se están dando en estos años. De acuerdo a la ley de Educación $\mathrm{N}^{\circ} 28044$, en el artículo $55^{\circ}$, se indica que un directivo debe ser visto "como un líder pedagógico que es el encargado de promover, conducir e interiorizar en los actores de la comunidad educativa el objetivo de lograr aprendizajes de los niños, niñas y adolescentes". En este artículo, queremos describir los factores que inciden en el ejercicio de la labor directiva para determinar el estilo de pensamiento ideal que el directivo debe poseer, o que debe tratar de adquirir para que no existan contradicciones con el estilo de liderazgo que ejerce.
\end{abstract}

Palabras clave: Estilo de liderazgo, estilo de pensamiento, líder pedagógico, calidad educativa, aprendizaje.

\begin{abstract}
At present, Peruvian education has as its main objective that all children and adolescents can learn. This consists in developing their skills, abilities and, therefore, their competences so that they can face the difficulties that arise in their daily lives. To this end, the Ministry of Education (Minedu onwards) has implemented strategies, and its main allies are teachers and managers within schools. The manager plays a very important role, since it will depend on whether or not the objectives proposed for the academic year are achieved. Before, managers were considered only as administrative agents, away from the pedagogical part. However, today our educational system has placed them as pedagogical leaders, so that they are direct participants in the educational reforms that are taking place in these years. According to Education Law No. 28044, in article $55^{\circ}$, it is indicated that a director must be seen "as a pedagogical leader who is responsible for promoting, conducting and internalizing in the actors of the educational community the objective of achieving learnings of children and adolescents ". In this article, we want to describe the factors that influence the exercise of managerial work to determine the ideal thinking style that the manager must possess, or that he must try to acquire so that there are no contradictions with the leadership style he exercises.
\end{abstract}

Keywords: Leadership style, thinking style, pedagogical leader, educational quality, learning.

\section{Fundamentos teóricos}

Para los fines del presente estudio, debemos precisar la diferencia entre la aptitud y estilo, de acuerdo a Sternberg (1999, p. 24) "un estilo es una manera de pensar. No es una aptitud, sino más bien una forma preferida de emplearlas aptitudes que uno posee. La distinción entre estilo y aptitud es fundamental. Aptitud se refiere a lo bien que puede hacer algo. Estilo se refiere a cómo le gusta a alguien hacer algo". El estilo de pensamiento se refleja en cómo actúan las personas ante alguna dificultad o problema; es decir, que los estilos de pensamiento son las formas que se eligen para hacer o afrontar algo. De acuerdo a la Teoría de Sternberg del "Autogobierno mental" (1997), (Sterngber (s/f); Yánez (2018); Nuñez et al(2005); Valadez (2009)) la sociedad es un reflejo de las diferentes formas de cómo el individuo se autogobierna y elige acciones ante situaciones o problemas que le surge durante su vida. Entonces, ¿qué ocurre cuando el estilo de liderazgo que debe poseer el directivo difiere del estilo de pensamiento que posee?.

En las Instituciones Educativas, ocurre que los directivos, a pesar de reconocer y ejercer un liderazgo apropiado, dejan que sus acciones se vean influenciadas principalmente por un particular estilo de pensamiento, lo cual ocasiona rupturas en las relaciones sociales creando un ambiente inestable en el trabajo.

\section{Liderazgo}

Los líderes pedagógicos son agentes educativos pilares del logro de objetivos que influyen directamente en la educación de todos los estudiantes. Para ello, los directivos necesitan tener un perfil de liderazgo que cree un ambiente, donde los docentes estén dispuestos a trabajar en un clima de confianza y donde las relaciones humanas sean positivas para el logro de las metas trazadas.

Se han desarrollado, a través de la historia, distintos tipos de teorías de liderazgo, como la Teoría de los Rasgos de Gordon Allport (1936), Teoría de la conducta o comportamiento, estudio por la Universidad de Michigan $(1942$ - 1957) y los estilos de liderazgo elaborados por Lewin (1939), Teorías basadas en el uso de la autoridad de Likert (1961), Teoría situacional o de contingencia de Paul Hersey y Kenneth Blanchard (1979) y la Teoría de las relaciones desarrollada por Burns (1978), basado en Castaño, (2013), Gomez (s/f), Guerra, (2018), Lapo, (2015), Sánchez, 2009).

Este artículo se centrará en dos tipos de liderazgo, que consideramos que son importantes que los directivos manejen en su relaciones interpersonales con sus 
empleados, y además porque son las teorías que manejan la mayoría de directivos y docentes, como veremos más adelante.

\section{Según la Teoría de la conducta o del comportamiento.}

a) Liderazgo centrado en las tareas, es cuando el líder se centra en el logro de los objetivos, es decir en las tareas que necesita realizar para alcanzar dicho objetivo, utilizando a sus sub alternos como herramientas para el logro de ello.

b) Liderazgo centrado en las personas, se da cuando el líder se centra en las necesidades de sus trabajadores y los estimula, los hace parte de las decisiones, toma en cuenta sus participaciones, para alcanzar los logros propuestos en su centro de trabajo.

\section{Estilos de Liderazgo}

Los estilos de liderazgo que ejerce el directivo para alcanzar los objetivos institucionales, como son los logros de aprendizaje, los cuales son evaluados en la ECE (Evaluación Censal de Estudiantes), que se dan en los grados de primaria y secundaria. .se observan en las relaciones directivo-docente.

Kurt Lewin (1939, citado por Alvarado, 2003, Castaño, 2013, Zuzama, 2015) dividió las relaciones líder-trabajador, en tres estilos:

a) Liderazgo autoritario o autocrático, en la cual la relación que existe entre jefe y empleado es unidireccional.

b) Liderazgo democrático, es cuando el líder crea un vínculo de trabajo en donde hay una relación de confianza entre él y sus trabajadores, haciéndole participes de las decisiones que se toma y valorando el trabajo que realizan para alcanzar las metas.

c) Liderazgo liberales o laissez faire, donde el líder deja todas las decisiones a los trabajadores, solo involucrándose en ellos, si es que se lo piden.

\section{Estilos de pensamiento}

Esta idea se desprende de la Teoría de Sternberg (1997), del "autogobierno mental", donde se explica que la sociedad es un reflejo de las diferentes formas de como el individuo se autogobierna y elige acciones ante situaciones o problemas que le surge durante su vida. Es decir, las personas ante alguna dificultad o problema va relacionar la inteligencia que posee con los conocimientos adquiridos que tiene; en ese actuar se podrá observar los estilos de pensamiento que maneja. Entonces, los estilos de pensamiento son las formas que se eligen para afrontar o hacer algo.

De acuerdo a Sternberg, los estilos de pensamiento son "aspectos asociados a las preferencias individuales por la ejecución de tareas, el desarrollo de proyectos y procesos mentales." (Citado por Jaimes, 2011, pp.3839).

Las funciones de los estilos de pensamiento "están referidas al tipo de labor que las personas desempeñan en el trabajo o en cualquier actividad diaria como preferencia por tareas, proyectos o situaciones, para adaptarse a su medio socio-cultural" (Escurra y otros, 2001 , p. 10); es decir, los individuos, para organizar las tareas que realizan de acuerdo al tipo de labor que desempeñan, van a ejercer determinadas funciones.



Fuente: Elaboración propia basada en Núñez (2005; pp. 3702, 3703)

Asimismo, existen cuatro formas del pensamiento, que determinan la forma en que el individuo "aborda" el mundo para solucionar problemas y realizar tareas:

a) Monárquico, se da cuando el individuo, se centra en una tarea o meta a la vez y utiliza cualquier medio para poder resolver los problemas que tiene.

b) Jerárquico, persona que se orienta a la resolución de múltiples tareas a la vez, organizándolas cada una con una prioridad concreta. Además es organizado cuando resuelve problemas y toma decisiones.

c) Oligárquico, se encuentra motivado a desarrollar múltiples tareas, que tienen el mismo rango de prioridad. Tiene o crea confusiones al momento de elegir las tareas.

d) Anárquico, es aquel individuo que se motiva por metas que le es difícil de clasificar.

Asimismo, rechaza las reglas, normas y procedimientos típicos (Núñez, 2005, pp. 3704, 3705).

Sternberg, por su parte, expone dos niveles, en los cuales el individuo va a aplicar sus habilidades cognitivas. Es decir, como el sujeto va a plantear una solución a un problema. a) Local, es cuando el individuo se enfoca en los aspectos concretos del problema o tarea. Prefiere trabajar en los detalles. b) Global, la persona se centra en el conjunto total del problema. No presta atención a los detalles. Prefiere tratar cuestiones abstractas (Núñez, 2005, p. 3705).

En otra dimensión de la Teoría del "Autogobierno mental", se mencionan dos alcances del pensamiento según el tipo de interacción que se tiene con otras personas:

a) Interno, el individuo prefiere trabajar solo, intentando resolver los problemas sin ayuda de los demás. Suele ser introvertido, reservado y centrado en las tareas.

b) externo, le gusta trabajar en equipo, son extrovertidos y están centrados en las personas más que en las tareas. (Núñez, 2005; pp. 3706, 3707).

Por último, Sternberg (1999), distingue dos inclinaciones del pensamiento, donde se observa la forma de ser respecto a la tendencia al cambio o novedad: a) Liberal, al sujeto le gusta enfrentar los cambios de diversas formas, hace cambios en su vida y rechaza lo estructurado. Asimismo, le gustan las situaciones de incertidumbre. b) Conservador, prefieren solucionar las 
situaciones de forma tradicional sin tomar riesgos. Prefiere situaciones claras y precisas (Núñez, 2005, p. 3707).

A partir de esta síntesis teórica, se puede afirmar que los estilos de pensamiento que deben poseer los líderes pedagógicos causan un impacto en la toma de decisiones, en las acciones y reacciones que estos tengan frente a una dificultad. Un directivo que tenga una función legislativa podrá innovar y crear junto a sus docentes contextos de aprendizaje para los estudiantes, asimismo tendrá la disposición de delegar actividades a las personas idóneas. De igual modo, si tiene una forma de pensamiento jerárquico, este podrá establecer prioridades respecto de las dificultades que enfrentará, distribuyendo recursos y siendo organizado, logrando, de este modo, las metas establecidas en la institución educativa.

Asimismo, el directivo que posea un estilo de pensamiento idóneo para el trabajo podrá optar y desarrollar con una facilidad el estilo de liderazgo apropiado para lograr las metas que se propone en su institución educativa, junto a sus trabajadores en un clima de confianza y buenas relaciones.

En el ámbito de educación, no se ha encontrado estudios referentes al directivo y los estilos de pensamiento. Sabemos que el directivo no solo debe contar con capacidades intelectuales sino también saber administrar esta capacidad para dirigir la inteligencia hacia la resolución de conflictos que se dan en la institución. Gonzales y otros (2011, p.140) indican que "En la práctica, también significa que no debemos entretenernos sólo en conocer el grado o nivel de las capacidades intelectuales, sino evaluar también la capacidad del sujeto para dirigir la inteligencia."

Asimismo, de acuerdo al estudio realizado por Sternberg y Gricorenko (1993), "la relación entre los estilos de pensamiento y el talento en donde se plantea la necesidad de considerar no solo las habilidades del talentoso sino también sus estilos de pensamiento para la identificación, instrucción y programación" (Citado por Caycho Rodriguez, 2009, p. 26). Por ende, podemos decir que es necesario y de suma importancia conocer los estilos de pensamiento de los directivos para poder alcanzar u obtener mejores resultados en la calidad educativa nacional.

Como se indica en el párrafo anterior, las investigaciones están dirigidas a relacionar los estilos de pensamiento con la inteligencia desde una perspectiva psicológica. Sin embargo, considero conveniente desarrollar estudios dedicados a los estilos de pensamiento en los directivos, para que se pueda formar un perfil idóneo y alcanzar o desarrollar contextos de clima favorable para los trabajadores, acoplándolo junto al estilo de liderazgo ideal para poder alcanzar el desarrollo de sus instituciones y obtener una calidad educativa que beneficiaría principalmente a los estudiantes en sus logros de aprendizajes, para que ellos puedan aplicarlo en su vida diaria.

Puntualicemos que los aprendizajes se logran en el aula y fuera de esta, para ello el docente debe de crear contextos de aprendizaje y para que esto ocurra, debe contar con un buen clima laboral. Por ello, es tan importante la función y el estilo de liderazgo y de pensamiento que este posea.

Además, creo que el reconocimiento de su propio estilo de pensamiento va a crear el interés en el directivo para poder fortalecer y cambiar algunos aspectos de su liderazgo. Jaimes (2011, p. 38) afirma lo siguiente "el conocimiento de los estilos de pensamiento propicia en los alumnos la investigación y el interés sobre sus propios procedimientos para enfrentar situaciones novedosas pues éste se motiva, al darse cuenta que hay ciertas tareas que se le van facilitando; fortalece su tolerancia, acepta la diversidad de estilos, crea una atmósfera adecuada que fomenta las actividades para estimularse como individuo, para desarrollar su potencial y enriquecerlo con nuevas experiencias que le amplíen su probabilidad de utilizar adecuadamente su intelecto".

\section{Ejercicio del estilo de liderazgo}

Los directivos han tenido capacitaciones sobre liderazgo pedagógico, convocados por el Minedu, Diplomado en Gestión Escolar y Segunda Especialidad en Gestión Escolar con Liderazgo Pedagógico, en la Universidad Peruana Cayetano Heredia - Facultad de Educación (2017). Sin embargo, en las instituciones educativas, el directivo ejerce no solo la función de líder pedagógico sino también de gestor administrativo. Ante esto, el líder se ve expuesto a situaciones en las que la toma de decisiones determina el alcance o no de los objetivos trazados.

En la realidad, ¿qué sucede cuándo el directivo toma decisiones? ¿Actúa conforme a lo que indica la teoría o predomina el estilo de cómo resuelve habitualmente las tareas?.

De acuerdo a las entrevistas que se realizó a docentes en base a su experiencia laboral, damos a conocer con ejemplos los estilos de liderazgo que se dan en las instituciones educativas. Para la docente D.H. que tiene 15 años laborando como docente, el directivo toma en cuenta las opiniones de los docentes, en algunas ocasiones, en las reuniones, siempre y cuando esté dirigido a lo que él cree conveniente. Sino de otra forma lo conduce hacia lo que él desea.

Asimismo, la docente S.G., que trabaja como docente hace 7 años, refiere que tuvo como experiencia en uno de sus centros de labores a un directivo, que realizaba las reuniones solamente para comunicar lo que él había decidió, sin tomar en cuenta las opiniones de los docentes.

Teniendo una mala relación con ellos, haciendo que estos no renueven contrato a final del año escolar, por el mal clima laboral que existía dentro de la institución educativa En ambos ejemplos, podemos deducir que el directivo toma un liderazgo autoritario, centrado en las tareas. Es decir que para lograr los objetivos, él opta por tomar las decisiones por sí solo, al final, si no encuentra apoyo de sus docentes.

Para el profesor F.M., que cuenta con 14 años de labor docente, hace una comparación entre dos instituciones en las cuales laboró, en una de ellas, la sub directora, trabajó un plan de mejora con los docentes, para lograr un mejor rendimiento en los resultados de la ECE, elaborando compromisos con los profesores, afianzando y reforzando las cualidades de cada uno de ellos, para designar un área para reforzar a los estudiantes, obteniendo un mejor resultado; a comparación del subdirector, en otra institución, que dejo enteramente la responsabilidad a sus docentes, solamente estimulándolos en una reunión a dar lo mejor de ellos. Por ende, podemos concluir que el directivo que planificó el plan de mejora, trabajo junto a sus docentes motivándolos continuamente, y resaltando sus fortalezas, tiene un estilo de trabajo basado en las personas y un estilo de liderazgo democrático. Por lo contrario, el último subdirector, utilizó un estilo de liderazgo liberal, delegando o dejando toda la 
responsabilidad a los docentes, a pesar de ver que al inicio preparó una reunión para solo dar a conocer el examen de ECE y que se debería de mejorar los resultados a comparación de los resultados obtenido el año anterior.

Un hecho que sucedió en dos distintas instituciones en las que he trabajado, es que en los meses de invierno, usualmente los patios se llenan de agua y los estudiantes no pueden salir a recrearse. En el primer colegio, la subdirectora, armó un plan de contingencia a partir de las experiencias en años anteriores, conversó con los docentes para concientizarlos y ser parte de las soluciones. Dentro del plan la líder juntó a sus docentes, realizaron distintas asambleas donde sensibilizaron a los padres de familia y lograron comprar un toldo para colocar en los patios, con las diferentes actividades que realizaron. En otra institución, el subdirector, a pesar de que las docentes le comentaban sobre la dificultad que había todos los años, no desarrolló ningún plan y en el momento que se dio la situación por la lluvia invernal, mandó a través de un memorándum, que las docentes se deberían quedar dentro del aula en hora de recreo para prevenir accidentes, creando malestar en los profesores y alumnos, no tomando en cuenta lo manifestado por ellas a inicios del año escolar.

A partir de estos casos, podemos conjeturar que la subdirectora ejerce un tipo de liderazgo, donde toma en cuenta sus docentes, formando una relación de confianza y empatía con las necesidades que tienen ellas, es decir un tipo de liderazgo centrado en las personas, y democrático. Por el contrario, la decisión del subdirector, produjo malestar a los alumnos y docentes. Por ello, podemos decir que tiene un liderazgo centrado en las tareas y es autoritario. En estos dos casos, podemos observar que el estilo de liderazgo de los directivos es totalmente contrario, a pesar de que ambos se han capacitado en liderazgo.

Influencia del estilo de pensamiento en el ejercicio del liderazgo

Del caso señalado anteriormente, podemos decir que el estilo de pensamiento para resolver las tareas que tuvo la subdirectora está basado en una función ejecutiva, ya que creó un proyecto a largo plazo a partir de sus experiencias y tuvo un alcance en el pensamiento externo, ya que trabajó en coordinación con los docentes y padres. Por el contrario, el subdirector utilizó una función legislativa, ya que partió y creó su propia solución al problema, sin tener en cuenta lo dicho por las docentes, en este caso su alcance de pensamiento fue interno, ya que prefiere trabajar solo.

¿Por qué resolvieron los problemas de manera tan diferente? Porque los estilos de pensamiento, al momento de resolver las tareas, son propios de cada persona, se basan en su experiencia y en cómo se relacionan con los demás. Añadiendo que la persona, en este caso el directivo posee una capacidad inherente a él de cómo dirigir su inteligencia, para resolver los conflictos. Como hemos leído en las bases teóricas, los estilos de pensamiento varían en cuanto a las funciones, formas, niveles y alcances de pensamiento de cada individuo. Las experiencias que los directivos tengan a lo largo de su ejercicio deberían ayudaran a tomar las mejores decisiones para resolver los problemas que se les presenten.

La docente F.N., que cuenta con 7 años de experiencia como docente, comenta que durante su experiencia pedagógica se ha encontrado con diversos tipos de directivos que ante una situación parecida han dado soluciones totalmente diferentes, explica ella que en todas las instituciones hay diferentes opiniones y que depende del directivo, manejarlo de manera acertada para llegar a un acuerdo. Una subdirectora, en uno de sus primeros trabajos, trataba de lidiar con aquellas situaciones, debatiendo los temas controversiales en la reunión general para que se pueda llegar a un consenso en forma general. Por otra parte, el otro directivo, aunque trataba siempre de llevar una buena relación con la plana docente, no podía manejar las reuniones, puesto que este, al ver que había opiniones distintas a las de él, optaba por la votación, siguiendo las reglas, que por mayoría se toma las decisiones. Como podemos ver, los estilos de pensamiento que ejercieron los directivos, en el estilo de liderazgo aplicadas en las reuniones de docentes, que son habituales en los centros educativos, tuvieron distintas formas de solucionar una dificultad. En el primer caso, podemos referir que tuvo un alcance de pensamiento liberal puesto que creó nuevas formas de poder dar solución a la dificultad que era desarrollar normalmente la reunión, haciendo que todos sus docentes se sientan confortables con la decisión tomada. Lo contrario ocurrió en el segundo caso: el directivo usó un alcance de pensamiento conservador, solo siguiendo las reglas, sin importar si todos sus docentes quedaban conformes.

Ante esto podemos decir que la forma de resolución de conflictos de los directivos puede ser distinta pero válida. Aquí surgiría la pregunta ¿las dos formas de resolver el conflicto son válidas, pero cuál sería la óptima? El liderazgo de ambos fue democrático, porque se respetó en una las opiniones y las buenas relaciones humanas y en la otro, la mayoría de votos en un acuerdo. La diferencia que hubo fue el estilo de pensamiento que utilizaron para solucionar la dificultad, es decir la capacidad que tuvieron ambos para poder dirigir toda su inteligencia no solo hacia la solución del problema sino en que también se afiancen las relaciones de confianza con los miembros de la plana docente.

\section{DISCUSIONES}

De acuerdo a las entrevistas realizadas en esta investigación a directivos y docentes, una dificultad que los líderes pedagógicos poseen, es el no poder realizar un óptimo trabajo, debĺdo a que su labor está dividida entre la administración y lo pedagógico.

El directivo D.C. expone que: "La teoría dice que el Director debe ser un líder pedagógico, vamos a suponer $50 \%, 50 \%$ líder administrativo, pero lamentablemente es al revés con tantas normas, yo creo que le dedico un $80 \%$ administrativo y lo demás al pedagógico".

La gran dificultad que tienen los directivos es que deben no solo encargarse de la parte pedagógica sino administrativa. Por ello, debe llevar un liderazgo que le permita crear tiempo para el trabajo administrativo, sin descuidar el trabajo con los docentes.

En este artículo, hemos mostrado ejemplos de liderazgo que emplean los directivos ante una situación de dificultad, distintos a un liderazgo democrático, el cual saben ellos que es lo óptimo para desempeñar este tipo de cargo.

Se desprende de la investigación que a pesar de que el directivo conoce que es lo mejor para su institución educativa y las relaciones humanas que construya: de confianza, empatía y valoración entre él y la plana docente, son los mejores instrumentos para poder lograr las metas, ¿por qué no utiliza ese tipo de liderazgo en todas sus decisiones? Creemos o consideramos que la 
respuesta es que cada directivo como persona posee un estilo de pensamiento, que va a influir al momento de tomar las decisiones o crear las herramientas para la resolución de determinados problemas. Hay predominancia de esto debido a la carga administrativa que puede generar tensión por cumplir con las obligaciones que demanda la UGEL y el propio Minedu.

De acuerdo a los fundamentos teóricos revisados, los directivos deben de tener un perfil que les permita tener las capacidades o desarrollar las capacidades para tener un buen liderazgo en sus instituciones, para ello, es importante conocer los estilos de pensamiento de cada uno de ellos, ya que de esta manera ellos mismos serán conscientes de la forma que afrontan los conflictos que suceden en su centro de labores. Conocer el estilo de pensamiento que poseen les permitirá desarrollar otras estrategias ante situaciones nuevas, asimismo para motivarse y estimularse, fortaleciendo su tolerancia para poder desarrollar su potencial como líder, y adaptar sus capacidades a nuevas estrategias que le permitan utilizar su inteligencia para el bien común de la institución educativa.

Por ello, debemos de hacer hincapié en que la educación de ahora necesita a líderes comprometidos con el logro de los objetivos a través de buenas prácticas de liderazgo que conlleven a estar centrados no solo en las tareas sino en las personas, estableciendo una relación de confianza y valoración entre él y sus colegas, en este caso directivo-docentes, para poder alcanzar los logros que se han trazado como institución, conociendo su estilo de pensamiento para poder dirigir su capacidades a la resolución de los conflictos de una manera adecuada no solo para él sino para todo los miembros de la comunidad educativa.

Dentro de esta problematica desde mi punto de vista, sugiero que el directivo debe poseer un liderazgo democrático que permita crear un buen clima de trabajo, donde los docentes puedan trabajar en un contexto de confianza, empatía y reconocimiento. De esta manera, lograría que los docentes trabajen coordinadamente y logren los objetivos propuestos en el centro educativo.

Los directivos ante la situación de una dificultad, debe tener en consideración las opiniones de los docentes, haciendo que sean parte de la solución, de esta manera colocándolos en una posición de "ponerse la camiseta", de esta manera construyendo un clima de confianza y de trabajo en conjunto.

Asi mismo es necesario contar con investigaciones que proporcionen información sobre los estilos de pensamiento de los directivos, que ayuden a construir una escuela que cuente con las herramientas para lograr los objetivos trazados.

Se recomienda hacer investigaciones sobre el tipo de liderazgo y los estilos de pensamiento de los directivos, para que puedan conocer y optar los mejores estilos de liderazgo y desarrollar y potenciar el estilo de pensamiento de los directivos, para fomentar una calidad educativa en las escuelas.

El Minedu debería de proveer no solo de capacitaciones a los directivos en el área académica, sino también implementar capacitaciones de orientaciones psicológicas, donde ellos puedan tener las herramientas para poder potenciar sus habilidades y buscar mecanismo de regulación ante sus dificultades.

Por todo el analisis realizado concluyo que el estudio teórico de los estilos de pensamiento es importante para el ejercicio de liderazgo directivo. Si el directivo conoce el estilo de pensamiento que posee, podrá fundar estrategias para sí mismo y adaptar sus capacidad, valiéndose de su inteligencia, al momento que deba tomar decisiones ante determinadas situaciones, asimismo, podrá motivarse y estimularse, fortaleciendo su tolerancia, desarrollándose como un líder efectivo en la institución que labora, llegando a cumplir de este modo los objetivos que propone el Minedu hoy en día.

\section{REFERENCIAS}

Alvarado, O. (2003). Gerencia y marketing educativo Herramientas modernas de gestión educativa. Lima - Perú: Udegraf S.A.

Castaño, R. (2013). Un nuevo modelo de liderazgo por valores. Madrid - España: Universidad de Alcalá. Departamento de Economía y Dirección de E m p r e s a s. R e c u p e r a d o d e : https://ebuah.uah.es/dspace/handle/10017/20002

Caycho, T. (2009). Estilos de pensamiento en estudiantes de $5^{\circ}$ grado de secundaria según nivel socioeconómico. (Tesis de pregrado). Universidad Nacional Mayor de San Marcos. Lima - Perú. $\mathrm{R}$ e c u p e $\mathrm{r}$ a d o d e http://cybertesis.unmsm.edu.pe/bitstream/handle/c ybertesis/2563/Caycho_rt.pdf? sequence=1\&isAllo wed $=y$

Escurra, L.; Delgado, A., Quesada, M. (2001) Estilos de pensamiento en estudiantes de la U.N.M.S.M., Revista de Investigación en Psicología, Vol.4 No.1. $\mathrm{R}$ e c u p e r a d o d e file:///C:/Users/Home/Downloads/Estilos de pensa miento en estudiantes de la UNMSM.pdf

Gómez, C. (2016) Liderazgo: Conceptos, Teorías y Hallazgos relevantes. Cuadernos Hispanoamericanos de psicología. Vol. 2, núm. 2, p p. 61 - 77 . R e c u p e r a d o d e http://www.elmayorportaldegerencia.com/Libros/Li derazgo/\%5 B P D \% 5 D \% 20 L ibros \% 20 $\% 20$ Liderazgo.pdf

González, J.; Núñez, C.; González S.; Álvarez, L.; Roces, C.; González, P.; Bernardo, A.; Valle, A.; Cabanach, R..; Rodríguez, S.; Sales, P. (2004) Estilos de pensamiento: análisis de su validez estructural a través de las respuestas de adolescentes al Thinking Styles Inventory Psicothema, Psicothema. Vol. 16, núm. 1, 2004, pp. 139-148. Recuperado de https://www.redalyc.org/pdf/727/72716122.pdf

Guerra, L. (2018) Análisis de las teorías de liderazgo: Una propuesta metateórica. Revista de ciencias administrativas y económicas. Vol. 1, núm. 1, 2018, p p. $56-75$. R e c u p e r a d o d e https://www.researchgate.net/publication/3267621 85 Analisis_de las teorias de liderazgo hacia u na propuesta metateorica Analysis of the leade rship theories Towards a metatheorical proposal /link/5b625f1d0̄f7e9bc79a74fc9e/download

Jaimes, M. (2011). Estilos de pensamiento e inteligencia emocional en estudiantes de psicología de dos universidades públicas. (Tesis de maestría) Universidad Nacional Mayor de San Marcos. Lima $\mathrm{P}$ e r ú . R e c u p e r a d o d e http://cybertesis.unmsm.edu.pe/bitstream/handle/c ybertesis/1465/Jaimes_cm.pdf?sequence=1\&isAllo wed $=y$ 
Lapo, M.; Jácome, M. (2019) El liderazgo y su evolución histórica. Revista Empresarial, ICE-FEE-UCSG ,Vol. 9, núm. 4, octubre -diciembre, pp. 11-16. $\mathrm{R}$ e c u p e r a d o d e https://dialnet.unirioja.es/descarga/articulo/641972 8.pdf

Nuñez, J. y otros (2005). Teoría del Autogobierno Mental: Análisis de los supuestos teóricos en relación al aprendizaje y la enseñanza. Coruña, España. $R$ e c u p e r a d o d e : http://www.educacion.udc.es/grupos/gipdae/docu mentos/congreso/VIIlcongreso/pdfs/442.pdf

Sánchez, I. (2009). Estilos de dirección y liderazgo en las organizaciones Propuesta de un modelo para su caracterización y análisis. Cali - Colombia: Universidad del Valle. Recuperado de http://bibliotecadigital.univalle.edu.co/bitstream/10 893/10312/3/Estilos\%20de\%20direccion\%20y\%20 liderazgo.pdf

Sternberg, R. (s/f). Theory of Mental Self - Government: Thinking Styles. Recuperado de http://www.robertjsternberg.com/thinking-styles

Sternberg, R. (1999). Estilos de pensamiento. Claves para identificar nuestro modo de pensar y enriquecer nuestra capacidad de reflexión. Barcelona - España: Paidós Ibérica S.A.

Valadez, M. (2009) Estilos de aprendizaje y estilos de pensamiento: precisiones conceptuales, Revista de Educación y Desarrollo, 11. Octubre-diciembre de 2009. Universidad de Guadalajara. Recuperado de http://www.cucs.udg.mx/revistas/edu desarrollo/an teriores/11/011 Huizar.pdf

Yánez, P. (2018) Estilos de pensamiento, enfoques epistemológicos y la generación del conocimiento científico. Revista Espacios. Vol. 39 (No 51) Año 2018 . P á $\mathrm{g}$. 18 . https://www.researchgate.net/publication/3297586 71 Estilos de pensamiento enfoques epistemol ogicos_y_la generacion_del_conocimiento_cientifi co

Zuzama, J. (2015). Liderazgo: estilos de liderazgo según Kurt Lewin y análisis de un caso real. (Tesis de pregrado) Universitat de les Illes Ballears. Palma E s p a ñ a. R e c u p e r a d o d e http://dspace.uib.es/xmlui/bitstream/handle/11201/ 3638/Zuzama_Covas_Juana_Maria.pdf?sequence $=1$ 